OPEN ACCESS

Edited by:

Ananda Pascual,

Mediterranean Institute for Advanced

Studies (IMEDEA), Spain

Reviewed by:

Karen Stocks,

Scripps Institution of Oceanography,

University of California, San Diego,

United States

Sonya Legg,

Princeton University, United States

*Correspondence:

Cora Hörstmann

cora.hoerstmann@awi.de

Specialty section:

This article was submitted to

Ocean Observation,

a section of the journal

Frontiers in Marine Science

Received: 14 August 2020

Accepted: 31 December 2020

Published: 21 January 2021

Citation:

Hörstmann C, Buttigieg PL,

Simpson P, Pearlman $J$ and Waite AM (2021) Perspectives on Documenting

Methods to Create Ocean Best

Practices. Front. Mar. Sci. 7:556234.

doi: 10.3389/fmars.2020.556234

\section{Perspectives on Documenting Methods to Create Ocean Best Practices}

\author{
Cora Hörstmann ${ }^{1,2 *}$, Pier Luigi Buttigieg ${ }^{3}$, Pauline Simpson $^{4}$, Jay Pearlman ${ }^{5}$ and \\ Anya M. Waite ${ }^{6}$
}

${ }^{1}$ Ecological Chemistry, Alfred-Wegener-Institut, Helmholtz-Zentrum für Polar- und Meeresforschung, Bremerhaven, Germany, ${ }^{2}$ Department of Life Sciences and Chemistry, Jacobs University, Bremen, Germany, ${ }^{3}$ Helmholtz Metadata Collaboration, GEOMAR, Kiel, Germany, ${ }^{4}$ International Oceanographic Data and Information Exchange of the Intergovernmental Oceanographic Commission, Oostende, Belgium, ${ }^{5}$ Institute of Electrical and Electronics Engineers, Paris, France, ${ }^{6}$ Ocean Frontier Institute and Department of Oceanography, Dalhousie University, Halifax, NS, Canada

This perspective outlines how authors of ocean methods, guides, and standards can harmonize their work across the scientific community. We reflect on how documentation practices can be linked to modern information technologies to improve discoverability, interlinkages, and thus the evolution of distributed methods into common best practices within the ocean community. To show how our perspectives can be turned into action, we link them to guidance on using the IOC-UNESCO Ocean Best Practice System to support increased collaboration and reproducibility during and beyond the UN Decade of Ocean Sciences for Sustainable Development.

Keywords: ocean best practices, ocean observation, method documentation, interoperability, digital repository, metadata management

\section{INTRODUCTION}

The ocean community is facing ever-increasing methodological complexity in its efforts to meet global challenges and to further discovery. Fragmentation of methods and data across regions, nations, and disciplines inhibits effective collaboration and ocean observation (Brett et al., 2020). Fortunately, a new culture of open sharing of knowledge on the web is merging along with the FAIR principles (Wilkinson et al., 2016), leading to new forms of dialog in the science community.

While their scope of application is broad, the FAIR principles are strongly focused on data, but can equally apply to methods, standards, and protocols. Indeed, if data is to be reproducible, reusable, and interoperable, then methods themselves have to be FAIR. Simultaneously, the community is recognizing that there is a reproducibility crisis in science (Lithgow et al., 2017) and marine science is no exception to this rule (Lowndes et al., 2017). However, multiple initiatives are promoting new, structured, and persistent ways to share methods online (Teytelman, 2018).

The path to digitizing methods and making them FAIR is not trivial, but concrete steps towards more structured and machine-readable documentation are already being taken. Developing the skill to write high-quality methods can be especially helpful for those early in their career or new 
to a field (Bell, 2014). Further, method documentation itself should be harmonized to make it transparent and easily understandable across multiple practitioners; and lastly, methods should be made available in online, accessible, and machinereadable ways. Guidelines, templates, and (e-)protocols exist in other fields such as medical research (Weissgerber et al., 2016; Aerts, 2018), accompanied with emerging possibilities in knowledge sharing and training (Nurhas et al., 2018; Nti et al., 2020). To help address this for the ocean community, Hörstmann et al. (2020) recently published an Intergovernmental Oceanographic Commission (IOC) Manual and Guide on enhancing method documentation for increased FAIRness.

The guidance in Hörstmann et al. (2020) is acted upon by the deployment of technologies underpinning the IOC Ocean Best Practices System ${ }^{1}$ (OBPS). The OBPS was developed to host and interlink methodological documents of any kind (protocols, guidelines, standard specifications, etc.); and seeks to support continuous convergence of methods as they undergo community refinement to become "best practices." In this way, the OBPS has laid a foundation for global, cross-community federation of method archiving and system development. However, the effective use of any system of this kind strongly depends on how it is used. That is, the way in which we - as an interdisciplinary ocean community - write and structure our methodological documents will have a direct effect on the effectiveness of the design, and technical capacities, of archiving and dissemination systems.

It is our responsibility to document our methods in a reproducible and transparent way, to efficiently use available technologies. From this perspective, we discuss the value and meaning of a "quality" method document, and how we use better documentation to share our methods within the community. Further, we reflect on the current state and future directions of handling diverse methodological content for a global community, increasingly making use of advanced digital resources in an inclusive and ethical way.

\section{WHAT DOES IT MEAN FOR A METHOD TO BECOME A BEST PRACTICE (AND WHY SHOULD WE CARE)?}

A method can be considered a best practice when it consistently produces superior results over other methods with the same objective(Simpson et al., 2018; Pearlman et al., 2019). Of course, context is key; what is best given a particular level of resourcing or environment of application (among many other factors), may not be so when circumstances change. Further, having a potential best practice in itself does not guarantee utility to the wider ocean community - only when shared, cross-validated, and used in the creation of better practices beyond its original scope, can we truly become excited about high-quality methods.

On the aspect of context-dependence, the perspective on what makes a method "best" can rapidly change across communities, disciplines, and resource levels. This is especially true, as most

${ }^{1}$ https://www.oceanbestpractices.org/ communities see their methods as the "best" for their own needs. Creating "best practices" across these scales therefore needs an inclusive, transparent, and sustained set of processes to communicate why, when, and for whom a method is a best practice.

What practical steps can we take to systematically identify localized best practices and explore their value in the broader global community? At the very least, methods must be sustainably archived, discoverable and accessible online to play a role in contemporary systems. In addition, technologies that interlink methods, and the communities that champion them, are needed to cope with the scale of the challenge (Buttigieg et al., 2019). Once the link is made, communities have the opportunity to test and advance methods in order to determine under what circumstances they are "best."

The persistence, visibility, and version control described above support widespread testing and cross-validation, thus allowing multiple practitioners to determine where, when, and how a method can be a best practice. To accomplish this, those that are developing methods and those that are archiving and interlinking them with new technologies must enter a more intense dialog. Through this, systems serving methods to the ocean community can draw closer to their end-users. This exchange encourages method evolution including adaptation for new technologies.

\section{HOW CAN WE SUPPORT THE OCEAN COMMUNITY IN CREATING BEST PRACTICES?}

To bridge key communities, the first, feasible, and particularly impactful step in this process is simply the use of more structured methodological documentation to synchronize novel technologies with users' needs, allowing more rapid and transparent improvements of ocean practices. We contend that structured templates, clear and complete metadata, version control, as well as mechanisms to support convergence and interdisciplinary exchange are foremost among the community's needs.

Templated documentation promotes well-structured, reproducible, and, in some cases, machine readable best practices. However, to effectively support the user experience, templates - and the sometimes required additional workload which comes with populating the templates - need to be introduced in a systematic way (Alwazae et al., 2014). Developers can be supported through additional guidance and explanations of the rationale behind the conventions suggested. In accordance with this principle, the ocean community called for guidance on how to effectively use the OBPS templates and what would be a "best practice for best practices" (BP4BP; Hörstmann et al., 2020) in method documentation (Simpson et al., 2020). With this, we hope that submitters are supported in archiving and sharing their methods to enable community refinement and global harmonization more successfully.

A key component of method documentation is the provision of relevant metadata. Pearlman et al. (2019) to access information about method maturity, relevant societal values/goals, and 
accompanying datasets, while the BP4BP document provides guidance on how users and developers can leverage them. This guidance reinforces the completeness of a document's metadata, which promotes machine access and further interaction with the system's technologies, and, consequently, document discovery and reuse by the community.

With organized and trusted online method documentation in place, systems are able to couple secure archiving and traceable versioning. This ensures that community refinement, extensions, and hard- or software changes are traced through the evolution of a method. In addition to being self-contained and complete, methodological documents created using the same templates can be more rapidly - and in some cases automatically - compared. Rapid intercomparison can greatly enhance our community's capacity to rigorously assess a large body of methods and choose those that are most appropriate to their mission and capacities.

As improved guidance on methodological development emerges, we are increasingly able to globally coordinate ocean observation and action, which have not yet converged. However, the technologies in methodology management systems (like the OBPS) rely on detailed, well-structured documentation to provide researchers with useful interfaces and functions. We note that developing such documentation is a continuous process and thus welcome the communities' critique of the BP4BP. ${ }^{2}$

Harmonized documentation supports interdisciplinary understanding of methods and data, and offers the possibility for better coordination in ocean observation. Methods then progress from stand-alone elements into more globally standardized pieces of the puzzle for improving global understanding of ocean systems.

\section{WHY ARE ETHICS AND INCLUSIVENESS CENTRAL TO THE DOCUMENTATION OF METHODS AND BEST PRACTICES?}

The ocean is in crisis yet offers humanity immense resources for sustainable development; in this complex reality, the publicly funded scientific community has an ethical obligation to rapidly share and collaboratively evolve the best of its methods to secure a truly sustainable and healthy relationship with the planet's oceans. This is especially true in the face of a scientific reproducibility crisis (Lithgow et al., 2017), data leaks (Gibney and Van Noorden, 2013), and a publication bias towards positive results, resulting in wasted time and resources (Rothstein et al., 2005). Further, consideration of how new methods directly or indirectly impact communities - and including those communities in the development of such methods in a co-design process, - is often overlooked, despite being essential to build and maintain trust at the science-society interface (Achterberg et al., 2015). A prerequisite for this trust is the (re)use, recognition, and official/institutional crediting of open methods as valued scientific outputs to support those making necessary changes in

\footnotetext{
${ }^{2}$ https://docs.google.com/document/d/1ME3XwRmVyltMQTP GDebHPfSrssLbCFjDetC8QGrJ-M/edit?usp=sharing
}

our culture of marine science. However, the ethics of sharing know-how grow in complexity as more stakeholders - outside of science are engaged to investigate ever broader and more multifaceted ocean and societal phenomena.

Across marine science, there is growing multilateral motivation to contribute scientific insight and methodology to societal missions through local, national, regional, and global programs. Simultaneously, science is integrating more methods from other sectors to interface with policy, the private sector, local communities, and other stakeholder groups. Consequently, inter-sectoral methodological harmonization and exchange has become a priority. To this end, scientific missions will need to include multi-sectoral perspectives and interests in solutionoriented research, and not only on the level of post-mission data transfer (Weichselgartner and Kasperson, 2010). Including such partners early, during method development itself, and working with them to balance the interests in and impacts of marine science will be a key strategy to promote regional sensitivity and trusted engagement across societal groups. Even if such groups cannot be immediately engaged, Hörstmann et al. (2020) describe how a document's content and prose may be selected, structured, and written to allow authors to engage with global stakeholders, which will be especially important to furthering the goals of the UN Decade of Ocean Science for Sustainable Development (henceforth, the "Ocean Decade"; Ryabinin et al., 2019).

An inclusive confluence of stakeholders and interests is a complex space; while it brings much-needed diversity to science, it also brings together different ethical norms that may not always align. In methodological development, this is often expressed through debates on what can be considered a "best practice" or even an appropriate way to conduct research, where, when, and by whom (Barbier et al., 2018). Discussion of these ethical challenges is broader than this article's scope, but we can take a concrete step towards improvement by proactively accounting for the ethical dimensions of any scientific method in its documentation. This is particularly important when a method impacts those who typically lack a voice in the scientific enterprise, to prevent the scientific discourse on standards, methods, and best practices being dominated by the larger or well-resourced global campaigns and actors, and high-level decision-makers.

In practical terms, we must co-develop a more consistent and transparent way to document the ethical dimensions of our methodologies. First, this will necessitate that ethical codes and guides (e.g., EU ethic appraisal procedure ${ }^{3}$ ) relevant to the marine sciences are digitized and made available online, following the same FAIR-aligned practices that systems like the OBPS use for other documents (e.g., DOIs, semantic indexing, searchability). Second, the sections of those methods where ethical concerns arise should consistently reference and/or link to such digitized ethical guidance enabling both humans and machines to readily understand when and where it is applicable.

\footnotetext{
${ }^{3}$ https://ec.europa.eu/research/participants/docs/h2020-funding-guide/crosscutting-issues/ethics_en.htm
} 
While the above will provide a path to build new networks of trust, ethical inclusion extends well beyond the inclusion of ethics in scientific practices. Rather, it sets a precedent whereby the co-development of methodology in marine science includes dedicated sections of methodological documents co-authored by other stakeholders (e.g., policy makers, citizen scientists, private sector interests, and conservation groups). This is a natural act of reciprocity to the same stakeholders who include scientific content and insights in their working documents, and will help address the persistent distrust in science across multiple sectors (Pechar et al., 2018). Further, such co-authored sections will be more broadly understandable to all groups involved, and connect them to resources and initiatives, which are of immediate relevance to their collective decision-making processes. The links and conventions which emerge from such inclusive interaction are a precursor to the application of technologies (referenced above) that will accelerate innovation, responsiveness, and crossboundary collaboration in best practice development for the marine sciences.

\section{CONCLUDING REMARKS AND THE PATH AHEAD}

The core of this perspective is that improved communication of methods, within and beyond marine science, is essential to interdisciplinary, community-led efforts to create a global collection of best practices. This evolves our responsibility to document our methodologies to support reproducibility into an opportunity to create an inclusive, global community of practitioners testing, adopting, and refining scientific methods in more diverse contexts.

Of course, the scale of this challenge needs a range of technologies to help our community coherently develop their methodologies. This perspective explored key aspects of increasing human-human and human-machine interaction, cross-linking documents, guidance, and information to support sustainable ways for truly inclusive and ethical method documentation.

To move forward, our community will be guided by a number of open questions that we collaboratively need to address:

- What are the major steps we need to take to document our methods for more universal understanding? We believe that structured templates and guidance documents, such as the BP4BP, are important steps in this direction, but the marine science community will need broader and continuous discussion of this theme to scope our needs and track advancements.

- Is our marine science community ready to accept more prescribed ways of documenting their methods? The diversity of needs within the community is high, with many overlaps and redundancies. Addressing this diversity should not compete with more standardized ways of documenting methods. Rather, the freedom of scientific exploration should be expanded through the improved communication structured documentation can bring, especially when combined with technologies able to enhance transparency and inclusivity. Balancing standards and innovation will be a persistent challenge in this space.

- How can we connect communities with similar methods and promote? Interoperable documentation is, again, a first step, but harmonization is a much more faceted, community-led process. We need a more systematic understanding of what motivates and incentivizes marine scientists to harmonize their methods, so that systems like the OBPS can more effectively accelerate it.

- How can we federate best practice development in marine science? We believe that systems that allow projects, programs, and other short-term initiatives to preserve, organize, and track the re-use of their methodological know-how can be federated to create a sustainable, global pillar of marine science. Interoperably cross-linking the holdings of regional and global platforms will unite distributed and independent systems already moving towards open, version-controlled, and transparent method sharing (e.g., Marine Sampling Field Manuals ${ }^{4}$ and the OBPS). Once again, how these systems and the community of practitioners contributing to them can be incentivized to build a common vision for a truly global solution is still to be discovered.

We invite the ocean community to join us in addressing these questions to continuously and collaboratively improve our collective methodological capacity. Through this, we will be able to unite and observe the ocean on larger scales than ever before. Together, we can evolve marine science into a planetary-scale and multi-stakeholder enterprise contributing to societal goals particularly the pursuit of a healthy marine ecosystem - with unparalleled coordination.

\section{AUTHOR CONTRIBUTIONS}

$\mathrm{CH}$ and PLB wrote the manuscript. CH, PLB, PS, JP, and AMW conceptualized the content and contributed to the manuscript.

\section{FUNDING}

$\mathrm{CH}$ was supported under the POF IV Research Programme topic 6 and subtopic 6.2 of the Alfred Wegener Institute Helmholtz Center for Polar and Marine Research. PLB's contributions were supported by the Helmholtz Frontiers in Marine Arctic Monitoring (FRAM) Programme of the Alfred Wegener Institute and the Helmholtz Metadata Collaboration (HMC). JP was supported by the US National Science Foundation through Grant 2500-1710-00 to the OceanObs Research Coordination Network. Funding for this publication was provided by the Ocean Frontier Institute, through an award from the Canada First Research Excellence Fund.

\footnotetext{
${ }^{4}$ https://introduction-field-manual.github.io/updates-and-revisions
} 


\section{REFERENCES}

Achterberg, P., de Koster, W., and van der Waal, J. (2015). A science confidence gap: Education, trust in scientific methods, and trust in scientific institutions in the United States. Public Underst. Sci. 26, 704-720. doi: 10.1177/ 0963662515617367

Aerts, J. (2018) From machine-readable CDISC Standard Specifications to the eProtocol (Conference Paper S109). PhUSE EU Connect 4th-7th November 2018, Frankfurt, Germany

Alwazae, M. M. S., Perjons, E., and Kjellin, H. (2014). "Quality measures for documentation of best practices," in Proceedings of the 47th Hawaii International Conference on System Sciences, (Honolulu, HI: University of Hawai'i at Mānoa), 3410-3419. doi: 10.1109/HICSS.2014.423

Barbier, M., Reitz, A., Pabortsava, K., Wolfl, A. C., Hahn, T., and Whoriskey, F. (2018). Ethical recommendations for ocean observation. Adv. Geosci. 45, 343-361. doi: 10.5194/adgeo-45-343-2018

Bell, J. (2014). Doing Your Research Project: A guide for first-time researchers, 6 Edn. Maidenhead: McGraw-Hill Education.

Brett, A., Leape, J., Abbott, M., Sakaguchi, H., Cao, L., Chand, K., et al. (2020). COMMENT: ocean data need a sea change to help navigate the warming world. Nature 582, 181-183. doi: 10.1038/d41586-020-01668-Z

Buttigieg, P. L., Simpson, P., Caltagirone, S., and Pearlman, J. S. (2019). "The ocean best practices system - supporting a transparent and accessible ocean," in IEEE. OCEANS 2019 MTS/IEEE SEATTLE, (Seattle, WA: IEEE), doi: 10.23919/ OCEANS40490.2019.8962680

Gibney, E., and Van Noorden, R. (2013). Scientists Losing Data at a Rapid Rate. London: Nature, doi: 10.1038/nature.2013.14416

Hörstmann, C., Buttigieg, P. L., Simpson, P., Pearlman, J., and Waite, A. M. (2020). Towards a Best Practice for Developing Best Practices in Ocean Observation (BP4BP): Supporting Methodological Evolution through Actionable Documentation. IOC Manuals Guides; 84. Paris: UNESCO, doi: 10.25 607/OBP-781

Lithgow, G. J., Driscoll, M., and Phillips, P. (2017). A long journey to reproducible results. Nature 548, 387-388. doi: 10.1038/548387a

Lowndes, J. S. S., Best, B. D., Scarborough, C., Afflerbach, J. C., Frazier, M. R., O'Hara, C. C., et al. (2017). Our path to better science in less time using open data science tools. Nat. Ecol. Evol. 1, 160. doi: 10.1038/s41559-017-0160

Nti, I. K., Adekoya, A. F., Opoku, M., and Nimbe, P. (2020). Synchronising social media into teaching and learning settings at tertiary education. Int. J. Soc. Media Interact. Learn. Environ. 6, 230. doi: 10.1504/ijsmile.2020.109228

Nurhas, I., De Fries, T., Geisler, S., and Pawlowski, J. (2018). "Positive computing as paradigm to overcome barriers to global co-authoring of open educational resources," in Proceedings of the 2018 23rd Conference of Open Innovations Association (FRUCT), (Bologna: IEEE), 283-292. doi: 10.23919/FRUCT.2018. 8588100
Pearlman, J., Bushnell, M., Coppola, L., Karstensen, J., Buttigieg, P. L., Pearlman, F., et al. (2019). Evolving and sustaining ocean best practices and standards for the next decade. Front. Mar. Sci. 6:277. doi: 10.3389/fmars.2019.0 0277

Pechar, E., Bernauer, T., and Mayer, F. (2018). Beyond political ideology: the impact of attitudes towards government and corporations on trust in science. Sci. Commun. 40, 291-313. doi: 10.1177/1075547018763970

Rothstein, H. R., Sutton, A. J., and Borenstein, M. (2005). "Publication bias in metaanalysis," in Publication Bias in Meta-Analysis: Prevention, Assessment and Adjustments, eds H. R. Rothstein, A. J. Sutton, and M. Borenstein (Hoboken, NJ: Wiley), 1-7. doi: 10.1002/0470870168.ch1

Ryabinin, V., Barbière, J., Haugan, P., Kullenberg, G., Smith, N., McLean, C., et al. (2019). The UN decade of ocean science for sustainable development. Front. Mar. Sci. 6:470. doi: 10.3389/fmars.2019.00470

Simpson, P., Pearlman, F., and Pearlman, J. (2018). "Evolving and sustaining ocean best practices workshop, 15 - 17 November 2017," in Proceedings of the AtlantOS/ODIP/OORCN Ocean Best Practices Working Group, (Paris: Intergovernmental Oceanographic Commission), 74. doi: 10.25607/OBP-3

Simpson, P., Pearlman, J., and Pearlman, F. (2020). Evolving and Sustaining Ocean Best Practices Workshop III. Oostende: IOC- IODE: GOOS and IEEE Oceanic Engineering Society, 37. doi: 10.25607/OBP-788

Teytelman, L. (2018). No more excuses for non-reproducible methods. Nature 560, 411. doi: 10.1038/d41586-018-06008-w

Weichselgartner, J., and Kasperson, R. (2010). Barriers in the science-policypractice interface: toward a knowledge-action-system in global environmental change research. Glob. Environ. Chang. 20, 266-277. doi: 10.1016/j.gloenvcha. 2009.11.006

Weissgerber, T. L., Garovic, V. D., Winham, S. J., Milic, N. M., and Prager, E. M. (2016). Transparent reporting for reproducible science. J. Neurosci. Res. 94, 859-864. doi: 10.1002/jnr.23785

Wilkinson, M. D., Dumontier, M., Aalbersberg, I. J., Appleton, G., Axton, M., Baak, A., et al. (2016). Comment: the FAIR guiding principles for scientific data management and stewardship. Sci. Data 3, 1-9. doi: 10.1038/sdata.2016.18

Conflict of Interest: The authors declare that the research was conducted in the absence of any commercial or financial relationships that could be construed as a potential conflict of interest.

Copyright (c) 2021 Hörstmann, Buttigieg, Simpson, Pearlman and Waite. This is an open-access article distributed under the terms of the Creative Commons Attribution License (CC BY). The use, distribution or reproduction in other forums is permitted, provided the original author(s) and the copyright owner(s) are credited and that the original publication in this journal is cited, in accordance with accepted academic practice. No use, distribution or reproduction is permitted which does not comply with these terms. 